\title{
A test statistic to quantify treelikeness in phylogenetics
}

$5{ }^{1}$ Division of Ecology and Evolution, Research School of Biology, Australian National University,

6 Canberra, Australian Capital Territory, Australia

$7 \quad{ }^{2}$ Research School of Computer Science, Australian National University, Canberra, Australian Capital

8 Territory, Australia

9 *Author for Correspondence: email: caitlin.cherryh@anu.edu.au

10 Key words: phylogenetic inference, model violation, systematic bias 


\section{Abstract}

12 Most phylogenetic analyses assume that the evolutionary history of an alignment (either 13 that of a single locus, or of multiple concatenated loci) can be described by a single 14 bifurcating tree, the so-called the treelikeness assumption. Treelikeness can be violated by 15 biological events such as recombination, introgression, or incomplete lineage sorting, and 16 by systematic errors in phylogenetic analyses. The incorrect assumption of treelikeness may 17 then mislead phylogenetic inferences. To quantify and test for treelikeness in alignments, 18 we develop a test statistic which we call the tree proportion. This statistic quantifies the 19 proportion of the edge weights in a phylogenetic network that are represented in a 20 bifurcating phylogenetic tree of the same alignment. We extend this statistic to a statistical 21 test of treelikeness using a parametric bootstrap. We use extensive simulations to compare 22 tree proportion to a range of related approaches. We show that tree proportion successfully 23 identifies non-treelikeness in a wide range of simulation scenarios, and discuss its strengths 24 and weaknesses compared to other approaches. The power of the tree-proportion test to 25 reject non-treelike alignments can be lower than some other approaches, but these 26 approaches tend to be limited in their scope and/or the ease with which they can be 27 interpreted. Our recommendation is to test treelikeness of sequence alignments with both 28 tree proportion and mosaic methods such as 3Seq. The scripts necessary to replicate this study are available at https://github.com/caitlinch/treelikeness

\section{Introduction}

32 A phylogenetic tree is a representation of the relationships between species or individuals. 33 Many estimates of phylogenetic trees implicitly assume that sites in a sequence alignment 34 share the same evolutionary history and conform to a single bifurcating tree. This 35 assumption is called treelikeness. This concept was first introduced by Dress (1984) and was 36 first used to assess how well data fit a tree by Eigen et al. (1988). Perfectly treelike 37 alignments are likely to be rare not only due to noise such as sequencing or alignment error 38 but also because biological processes like incomplete lineage sorting (ILS), recombination, or 39 introgression mean even very short alignments may have an evolutionary history that 40 cannot be represented by a single bifurcating phylogeny (Mallet et al. 2016; Mendes et al. 41 2019; Scornavacca and Galtier 2017). Although the treelikeness assumption is almost 
42 universally made in phylogenetic analyses, it remains rare to test the validity of this

43 assumption. If treelikeness is incorrectly assumed, phylogenetic inferences may be misled

44 (Brown and Thomson 2018), so it is important to test whether the treelikeness assumption

45 holds prior to estimating a phylogenetic tree. Doing so may assist phylogeneticisits in

46 choosing the best combination of data and inference method with which to infer their

47 phylogeny.

49 Most estimates of phylogenetic trees assume that the data are treelike at some level.

50 Concatenation methods (also known as supermatrix methods) assume that all loci in an

51 alignment share a single evolutionary history. This approach has been criticised as the

52 histories of individual loci may vary dramatically, potentially resulting in incorrect

53 phylogenetic inferences (Shi and Yang 2018; Weisrock et al. 2012; Wielstra et al. 2014; Wu

54 et al. 2018; Zhao et al. 2016). Concatenating alignments from loci with different

55 evolutionary histories clearly violates the treelikeness assumption. Coalescent methods

56 improve on the concatenation method by explicitly incorporating non-treelikeness due to

57 ILS. To do so, coalescent methods allow each locus to have a separate tree topology and use

58 the distribution of these topologies to then infer the species tree under a model of ILS.

59 However, these methods still assume that the alignment used to produce each single-locus

60 tree is treelike. Recent work shows that different exons in the same gene often have

61 different evolutionary histories, meaning that in many cases the alignments used to

62 estimate single-locus trees may not be treelike (Mendes et al. 2019; Scornavacca and Galtier

63 2017). As a result, both concatenation and coalescent tree estimation methods may be

64 vulnerable to errors introduced by violation of the treelikeness assumption.

66 Previous studies have proposed a range of approaches to measuring certain aspects of non-

67 treelikeness. Goldman (1993) developed the first general test for model adequacy in 68 evolutionary models, which simultaneously assess all assumptions of the evolutionary 69 model, including the treelikeness assumption. Unfortunately, as all parameters of the model 70 are tested simultaneously, the treelikeness of the alignment cannot be extracted from the 71 results of this test. Additionally, several methods for visualizing treelikeness have been 72 suggested. Likelihood mapping (Strimmer and von Haeseler 1997) is primarily used to 73 visualise and estimate the phylogenetic signal of an alignment (Baric et al. 2003; Salzburger 
74 et al. 2002; Steiner and Dreyer 2003) but has also been used to assess whether an alignment

75 had a treelike structure (Nadan et al. 2003). The $\delta$ plot method (Holland et al. 2002) allows

76 assessment of the treelikeness of an alignment using a mathematical approach based on

77 assessing the treelikeness of all possible quartets of taxa in the alignment. The mean $\delta_{\mathrm{q}}$

78 value from the $\delta$ plot method has been used to draw inferences about the overall

79 treelikeness of an alignment, although the interpretation of this value varies: values of 0.11

80 (Kozak et al. 2015) and 0.28 (Short et al. 2014) have been suggested to indicate significant

81 non-bifurcating signal, and a value of 0.18 was suggested to indicate that an alignment was

82 treelike (Grimm and Renner 2013).

84 Phylogenetic split networks have also been used to visualise the treelikeness of an 85 alignment. A split network generalises a phylogenetic tree by representing incompatible 86 phylogenetic signals present in the sequence alignment as additional edges (Huson et al. 87 2010). Compared to a tree, a phylogenetic split network includes more information about 88 the relationships between taxa as it includes conflicting phylogenetic signals and alternate 89 histories (Bryant and Moulton 2004). The treelikeness of alignments can be visually 90 determined based on the number, size and position of parallelograms within the network 91 (Bryant and Moulton 2004; Kennedy et al. 2005; Kück et al. 2010). Phylogenetic networks 92 provide a very useful visual tool for assessing treelikeness, but they can be somewhat 93 difficult to interpret and there currently exists no framework for comparing the treelikeness 94 of different alignments using networks. Ideally, a test statistic should quantify treelikeness 95 in a way that is comparable across alignments and allow biologists to make informed 96 decisions about which data and methods to use for inferring evolutionary histories.

98 Here, we introduce the tree proportion for quantifying the treelikeness of a multiple 99 sequence alignment. The tree proportion of an alignment describes the proportion of a 100 phylogenetic network of that alignment that can be represented by a single bifurcating tree. 101 Specifically, it is the proportion of non-trivial split weights of an inferred network that are 102 contained in a bifurcating phylogenetic tree of the same alignment. A split is trivial if one 103 side of the bipartition contains only one taxon, i.e. terminal branches on a phylogeny 104 represent trivial splits, because they are contained in all trees and networks and thus 105 provide no information about treelikeness. Tree proportion ranges from 0 to 1 , where a 
106 score of 0 indicates that none of the non-trivial splits in the network are represented in the 107 tree. A score of 1 indicates that all of the non-trivial splits in the network are represented in 108 the tree (i.e. the alignment is perfectly treelike). More generally, the better that bifurcating 109 phylogenetic tree represents an alignment, the closer the tree proportion will be to 1.

111 In addition to providing an intuitive measure of treelikeness, we describe how the tree 112 proportion can be used to ask whether the assumption of treelikeness can be rejected for 113 any given alignment. To do this, we use a parametric bootstrap to simulate treelike datasets 114 with model parameters estimated from the original alignment, and then ask whether the 115 observed tree proportion is surprisingly small relative to the tree proportions observed from 116 the (truly treelike) simulated datasets. This allows us to generate a p-value for the test 117 statistic, where a p-value $<0.05$ indicates that the assumption of treelikeness can be 118 rejected for the alignment in question. Finally, using introgression as a framework for 119 simulating alignments of varying treelikeness, we demonstrate how the tree proportion can 120 be used to quantify and test for treelikeness, and we compare its performance to previously 121 suggested methods for estimating treelikeness, as well as certain measures that have been 122 suggested specifically for testing introgression.

\section{New approaches}

\section{Tree proportion}

126 Tree proportion is defined as follows. For a split network denoted by $(S, \lambda)$ where $S$ is the 127 set of non-trivial splits and $\lambda$ is a split weight function, and a phylogenetic tree $(T)$ the tree 128 proportion is calculated as:

$$
\text { Tree proportion }=\frac{\sum \sigma \in S \cap T \lambda(\sigma)}{\sum_{\sigma \in S} \lambda(\sigma)}
$$

130 In other words, tree proportion is the proportion of the total weight of non-trivial splits in 131 the network that are represented by the tree. Figure 1 illustrates how to calculate the tree 132 proportion for a simple five-taxon split network and tree.

134 Calculating tree proportion requires both a bifurcating phylogenetic tree and a phylogenetic 135 split network estimated from the same alignment. In principle the split network and the 
136 bifurcating phylogenetic tree could be inferred with any method. Indeed, the maximum tree

137 proportion for any given split network can be calculated simply from using the maximum

138 spanning tree of the network as the bifurcating tree. In this study, we used Maximum

139 Likelihood to estimate bifurcating trees for two reasons: (1) Maximum Likelihood is one of

140 the most commonly used methods for tree inference, and (2) Maximum Likelihood naturally

141 allows us to extend our approach to include a parametric bootstrap test because it co-

142 estimates the bifurcating tree and the parameters of a model of molecular evolution. We

143 used NeighborNet (Bryant and Moulton 2004) to estimate the split network. NeighborNet is

144 a distance based agglomerative method for generating replicable and statistically consistent

145 split networks. NeighborNet measures conflict rather than evolutionary history, so the

146 resulting network represents conflicting signals within the alignment.

148 To calculate the tree proportion of an alignment, we first estimated a NeighborNet network

149 in SplitsTree v4.14.6 (Huson and Bryant 2006). Next, we estimated a maximum likelihood 150 tree for the same alignment using IQ-Tree v2.0 with ModelFinder (Kalyaanamoorthy et al.

151 2017; Minh et al. 2020). Finally, we calculated tree proportion in R using code available from 152 https://github.com/caitlinch/treelikeness.

\section{Parametric bootstrap}

155 Because we do not assume any prior distribution of tree proportion, we rely on a parametric 156 bootstrap procedure to determine whether the tree proportion is significantly lower than 157 would be expected for truly treelike alignments as follows. For a given alignment $D$, we 158 reconstruct a maximum likelihood tree $T_{M L}$ with the best-fit substitution model $M$. From $159 T_{M L}$ and $M$ we simulate $n$ ( $\mathrm{n}=199$ by default) alignments $D_{1}, \ldots, D_{n}$. From each $D_{i}$ we 160 reconstruct a maximum likelihood tree $T_{i}$ and a NeighborNet network $S_{i}$ and use $T_{i}$ and $S_{i}$ 161 to calculate the tree proportion $T P_{i}$. We calculate the statistics $T P_{1}, \ldots, T P_{n}$. The $p$-value is 162 then computed as the fraction of $T P_{i}$ greater than or equal to $T P$ of the original alignment.

\section{Results}


166 Of the six test statistics for treelikeness we compared, tree proportion $\left(R^{2}=0.863\right)$ and

167 mean $\delta_{\mathrm{q}}\left(\mathrm{R}^{2}=0.818\right)$ showed the strongest correlations with the proportion of introgressed

168 DNA (Figure 2). Both tree proportion and mean $\delta_{\mathrm{q}}$ were strongly correlated with the 169 proportion of introgressed DNA whether the simulated introgression was reciprocal or non170 reciprocal (Supplementary Figure 1: Tree proportion $R^{2}=0.863$ and 0.704 ; mean $\delta_{q} R^{2}=$ 1710.818 and 0.902 ). Tree proportion was strongly correlated with the proportion of 172 introgressed DNA regardless of the simulated tree depth (Supplementary Figure 2, all $R^{2}>$

173 0.692). Mean $\delta_{\mathrm{q}}$ showed strong correlations on tree depths up to 0.5 (Supplementary Figure

1742 , all $R^{2}>0.705$ ) but a much weaker correlation when the simulated tree depth was 1.0 175 (Supplementary Figure $2, R^{2}=0.344$ ). The strength of the correlations between the 176 proportion of introgressed DNA and the other four test statistics was highly variable and 177 never higher than 0.511 under any simulation conditions (Figure 2, Supplementary Figures 1 178 and 2).

180 The ability of each test to statistically reject treelikeness under simulated introgression 181 events varied greatly (Figure 3). PHI and 3SEQ had the highest power to reject non-treelike 182 alignments, and the tests successfully detect $100 \%$ of introgression events after the 183 proportion of introgressed DNA reached 0.2 and 0.1 respectively (Figure 3). Mean $\delta_{\mathrm{q}}$ and 184 tree proportion have intermediate results. At a proportion of introgressed DNA of 0.5 , these 185 tests detect $98 \%$ and $99 \%$ respectively of the alignments containing introgression (Figure 3). 186 Proportion of resolved quartets and mode $\delta_{\mathrm{q}}$ failed as statistical tests and did not 187 successfully detect introgression events for any tree depth or event type (Figure 3, 188 Supplementary Figures 3 and 4). Test results were very similar regardless of whether 189 introgression was simulated as a reciprocal or a non-reciprocal event (Supplementary Figure 190 3). All test statistics except proportion of resolved quartets and mode $\delta_{\mathrm{q}}$ have acceptable 191 false positive rates (i.e., a significant result in approximately $5 \%$ of tests when there is no 192 introgression in the simulation, shown as 0 on the $x$-axis of each panel in Figure 3). Both 193 3SEQ and PHI have less power to detect introgression at lower tree depths. For a tree depth 194 of 0.05 substitutions per site and at a proportion of introgressed DNA of 0.5, PHI and 3SEQ 195 correctly identify $96 \%$ and 95\% of introgression events respectively (Supplementary Figure 196 4). Conversely, tree proportion has less power to detect introgression at higher tree depths. 197 For a tree depth of 1 substitution per site at a proportion of introgressed DNA of 0.5, tree 
198 proportion correctly identifies just over a third (35\%) of introgression events

199 (Supplementary Figure 4).

200

201

Decreased treelikeness due to increasing number of introgression events

202 Of the six test statistics we compared, only 3SEQ and tree proportion revealed clear 203 decreases in treelikeness as the number of introgression events increased (Figure 4).

204 Encouragingly, tree proportion responded similarly for all tree depths and whether or not

205 the simulated recombination was reciprocal or non-reciprocal (Supplementary Figures 5 and

206 6). 3SEQ test statistic values were similar across reciprocal and non-reciprocal

207 recombination events (Supplementary Figure 5), but the range of values is dependent on

208 tree depth (Supplementary Figure 6). The PHI test statistic only identified nonreciprocal

209 introgression events (Supplementary Figure 5), and was more strongly correlated to the

210 number of introgression events at higher tree depths (Supplementary Figure 6). The

211 proportion of resolved quartets, mean $\delta_{\mathrm{q}}$ and mode $\delta_{\mathrm{q}}$ test statistic values showed at best

212 weak correlations with the number of introgression events, regardless of the simulation

213 conditions (Figure 4; Supplementary Figures 5 and 6).

215 Three test statistics performed well as statistical tests to reject treelikeness in the presence

216 of multiple introgression events (Figure 5). 3SEQ, tree proportion and PHI had the highest

217 power to reject non-treelike alignments, as the tests successfully detected $100 \%$ of

218 alignments containing introgression after 1, 2, and 3 events respectively (Figure 5). These

219 results were similar for nonreciprocal and reciprocal events (Supplementary Figure 7). The

220 best performing test statistic was tree proportion, which behaved similarly for all event

221 types and tree depths (Supplementary Figures 7 and 8). 3SEQ performed well at tree depth

222 of 0.5 substitutions per site (Figure 5) but showed very poor performance at the lowest tree

223 depth of 0.05 substitutions per site (Supplementary Figure 8). PHI also performed well at

224 tree depth of 0.5 substitutions per site (Figure 5), but its performance dropped below that

225 of tree proportion at low tree depths (Supplementary Figure 8). The other three statistics

226 (proportion of resolved quartets, mean $\delta_{\mathrm{q}}$ and mode $\delta_{\mathrm{q}}$ ) were unable to reject treelikeness

227 for the simulated introgressed alignments at any tree depth or event type (Figure 5,

228 Supplementary Figures 7 and 8). Only PHI and tree proportion had acceptable false positive 
229 rates (i.e., a significant result in approximately $5 \%$ of tests when there is no introgression in

230 the simulation, shown as 0 on the $x$-axis of each panel in Figure 5).

232 Discussion

233 In this study, we introduce the tree proportion as a way of measuring treelikeness, and 234 testing (with a parametric bootstrap) whether a single bifurcating phylogenetic tree is 235 sufficient to explain the evolutionary history of an alignment. Importantly for a proposed 236 measure of treelikeness, tree proportion values are easy to interpret: a value of 1 237 corresponds to a perfectly treelike alignment (i.e. one whose evolutionary history can be 238 perfectly explained by a single bifurcating phylogenetic tree), and as treelikeness reduces 239 the tree proportion will decrease towards zero. These properties mean that the tree 240 proportion can be used to directly compare the treelikeness of different alignments.

242 We use a suite of simulations to compare the tree proportion to five other tests, both as a 243 measure of treelikeness and as a statistical test that can be used to reject treelikeness for a 244 given alignment. Our results show that the tree proportion is a very useful measure of 245 treelikeness: under a huge range of simulation conditions tree proportion consistently 246 declines in concert with declines in the treelikeness of the alignment. When used as a 247 statistical test to ask whether an alignment can reject treelikeness, the six test statistics we 248 compare have varied success at detecting multiple causes of decreased treelikeness, and no 249 one test performed the best across all simulation conditions. Tree proportion, 3SEQ (Lam et 250 al. 2018) and PHI (Bruen et al. 2006) performed well as statistical tests rejecting treelikeness 251 under simulated introgression events, and we found that at least one of these tests 252 detected almost every simulated recombinant alignment in the majority of cases. It is 253 perhaps unsurprising that PHI and 3SEQ performed well: both tests are designed to detect 254 recombinant sequences of exactly the type we simulated. However, introgression is just one 255 example of a biological event that reduces the treelikeness of an alignment, and our 256 simulations show that both PHI and 3SEQ performed poorly in certain simulation conditions.

257 As a result, we suggest that statistical tests of treelikeness for empirical alignments would 258 be best served by combining a phylogenetic approach such as tree proportion with a 259 mosaic-based test such as 3SEQ. The tree proportion test works in a wide variety of 
260 conditions, and produces an easy-to-interpret test statistic. However, in many conditions

261 (such as if the non-treelikeness is caused by introgression and the tree depth is above a

262 certain threshold) 3SEQ has much more power to detect non-treelikeness. Using both tests

263 therefore provides the most generality and power across all possible causes of non-

264 treelikeness that may impact phylogenetic analyses.

265

266 Tree proportion joins a growing group of tests for absolute model adequacy. Penny et al.

267 (1992) wrote that a fundamental criterion for a scientific method is that the data must be

268 able to reject the model, a requirement that is rarely met in phylogenetics (Brown and

269 Thomson 2018). Most phylogenetic analyses proceed with only relative tests of model

270 adequacy (such as ModelFinder (Kalyaanamoorthy et al. 2017) which selects the best model

271 from a pre-defined set of a models) or no test (Cui et al. 2013; de Souza et al. 2018; Grismer

272 et al. 2018; Grybchuk et al. 2018; Kang et al. 2014; Lei and Dong 2016; Pearce et al. 2017;

273 Tay et al. 2017). As model violation is widespread across phylogenetic datasets (Naser-

274 Khdour et al. 2019), phylogenetic analyses may benefit if absolute tests for model adequacy

275 are performed prior to tree estimation. Tree proportion builds on the absolute test for

276 model accuracy developed by Goldman (1993). While Goldman's test encompasses all

277 assumptions of the tree and model which are used to calculate the likelihood in

278 phylogenetic analyses, the tree proportion assesses a subset of model assumptions, and

279 asks specifically to what extent a single bifurcating tree is adequate for explaining the

280 evolutionary history of a given alignment.

281

282 The $\mathrm{PHI}$ test has been widely used to detect recombination in phylogenetic alignments

283 (Cabanne et al. 2008; Croll and Sanders 2009; Croucher et al. 2015; D'Horta et al. 2011;

284 Faria et al. 2016; Harris et al. 2012; Joly and Bruneau 2006; Ogura et al. 2009; Pinho et al.

285 2008; Tian et al. 2012; Weinert et al. 2009). The PHI test performed well in our simulations

286 and detected almost all introgression events under all simulation conditions. Similarly,

287 Bruen et al. (2006) and Haubold et al. (2013) found the PHI test accurately detected

288 recombination in simulated coalescent data and in empirical datasets including bacteria,

289 fungi and virus DNA, and animal mtDNA. However, the PHI test performs poorly when

290 sequence diversity is low (less than 10\%), when alignments are short and/or when the

291 number of taxa is low (less than 10) (Bruen et al. 2006; White et al. 2013; White and 
292 Gemmell 2009). In previous simulations where the power of the PHI test was low, the

293 sequence diversity ranged from 0.01 to $1.25 \times 10^{-3}$ (Bruen et al. 2006; White et al. 2013;

294 White and Gemmell 2009). The diversity in our simulated alignments was much higher than

295 this, and therefore our simulations contained sufficient informative sites and

296 incompatibilities for the test to perform well. The PHI test is a powerful and accurate test

297 for recombination when the sequence diversity and number of taxa are sufficiently large,

298 and is conservative and results in false negatives when they are not.

300 Likelihood mapping and $\delta$ plots allow visualisation of the phylogenetic content of a 301 sequence alignment, but we show here that they do not perform well as test statistics for 302 treelikeness. The mean $\delta$ value has been used to quantify the treelikeness of an alignment, 303 with values from 0 to 0.2 generally interpreted as highly treelike (Coiro and Barone Lumaga 304 2018; Dashper et al. 2017; Grimm and Renner 2013; Kozak et al. 2015; Meier-Kolthoff and 305 Göker 2019; Short et al. 2014; Stanborough et al. 2018). However, the mean $\delta$ responds 306 inconsistently to causes of decreased treelikeness, so a low mean $\delta$ value does not 307 necessarily indicate that an alignment is treelike (e.g., the mean $\delta$ value was 0.048 for one 308 of our simulations in which the alignment contained 8 introgression events). Similarly, the 309 proportion of resolved quartets from likelihood mapping has been interpreted as an 310 indicator of the treelikeness of an alignment, with values from 0.8 and up interpreted as 311 treelike (Buesa et al. 2002; Elena et al. 2001; Li et al. 2017; Morgan et al. 2014; Nadan et al. 312 2003; Pitra et al. 2002; Salemi et al. 2000; Shi et al. 2012; Verbruggen and Theriot 2008). 313 Likelihood mapping displays the phylogenetic content of an alignment by plotting the 314 treelikeness of individual quartets. However, our results show that this correlates very 315 poorly with the overall treelikeness of an alignment (e.g., the proportion of resolved 316 quartets was 1.0 for some of our simulations in which the alignment contained 8 317 introgression events). The proportion of resolved quartets for our simulations is high - the 318 minimum value was 70\% (Supplementary Figure 2), but the majority of simulations had a 319 proportion of resolved quartets above $85 \%$, despite most of them containing significant 320 non-treelikeness such as multiple introgression events involving a large fraction of the 321 alignment. The reason that the proportion of resolved quartets remains high in these 322 simulations is that each introgression event involves only a few taxa, meaning only a small 
323 proportion of quartets are affected and the contribution to the proportion of resolved

324 quartets is low. As a result of these limitations, we recommend using these methods for

325 visual assessment of phylogenetic information, but not for quantifying or testing for 326 treelikeness.

328 While the parametric bootstrap approach we propose here is designed to isolate non329 treelikeness from other signals in the data, this separation will not always be perfect. A 330 statistically significant result from the parametric bootstrap indicates a significant difference 331 in test statistic values between the original alignment and the bootstrap replicates. In our 332 simulations, the only difference between the original alignment and the bootstrap replicates 333 was that the former included introgression events. The same will not be true for empirical 334 alignments, as the models we use for empirical data are gross oversimplifications of the true 335 underlying process (e.g. (Song et al. 2010) (Lemmon and Moriarty 2004)). As a result, the 336 parametric bootstrap may return a significant result if other types of model violation lead to 337 differences in the treelikeness between the empirical and the bootstrap-replicate 338 alignment. Given that model violation is widespread and common within phylogenetic 339 datasets (Naser-Khdour et al. 2019), we suggest that a significant result from the parametric 340 bootstrap should be interpreted as likely, but not certain, to be caused by non-treelikeness 341 in the empirical alignment. Regardless of the cause, a significant result from the parametric 342 bootstrap should be cause for concern, and perhaps warrant further investigation of the 343 offending alignment.

\section{Materials and Methods}

\section{Simulation approach}

347 Introgression is a potential source of non-treelikeness that is known to mislead phylogenetic 348 inferences (Posada and Crandall 2002; Wiens 1998). However, it is hard to account for with 349 current methods. Introgression provides a framework wherein different types and amounts 350 of non-treelikeness can be simulated on a linear scale (Posada and Crandall 2002). In this 351 study, we use introgression as a framework for simulating non-treelike alignments in order 352 to compare new and existing measures and tests for treelikeness. Here, we compare tree 353 proportion with two tests for introgression: the Pairwise Homoplasy Index (PHI) (Bruen et 
354 al. 2006) and 3SEQ (Boni et al. 2007; Lam et al. 2018). We also applied two existing methods

355 that have been used to test for treelikeness in previous studies: $\delta$ plotting (Holland et al.

356 2002) and likelihood mapping (Strimmer and von Haeseler 1997).

$358 \mathrm{PHI}$ measures the minimum number of convergent mutations on any tree to describe the 359 genealogy of a pair of sites. $\mathrm{PHI}$ is a widely used test for recombination or introgression and 360 was previously found to outperform other similar tests (Bruen et al. 2006). We calculated 361 the PHI value and p-value for the each alignment using PhiPack (Bruen 2005).

363 The second test, 3SEQ, attempts to calculate the number and location of introgression 364 events for a given alignment by testing each triplet of sequences using a hypergeometric 365 random walk to determine if one sequence is the child of the other two (Boni et al. 2007; 366 Lam et al. 2018). 3SEQ has been shown to perform well in simulations (Boni et al. 2007; Lam 367 et al. 2018). We used the 3SEQ implementation (Lam et al. 2018) to calculate the number of 368 recombinant triplets, number of recombinant sequences and p-value for each alignment.

370 We also applied both the $\delta$ plotting (Holland et al. 2002) and likelihood mapping (Strimmer and von Haeseler 1997) methods, which have been previously used to estimate treelikeness

372 of an alignment. To obtain a test statistic for the $\delta$ plotting method, we applied the 373 delta.plot function in the R package ape v5.4-1 (Paradis et al. 2004) to the distance matrix 374 for each alignment and calculated the mean and mode $\delta_{\mathrm{q}}$ value. The mean or mode $\delta_{\mathrm{q}}$ will 375 be between 0 and 1, where larger values are less treelike. We used the likelihood mapping 376 implementation in IQ-Tree (Minh et al. 2020) with the number of quartets to sample set to 37725 times the number of taxa, and took the test statistic to be the number of fully-resolved 378 quartets. This test statistic is a proportion, with a value of 1 indicating that every quartet 379 sampled was treelike. The test statistic value decreases as the quartets become less treelike.

380 The p-values for both the $\delta$ plotting and likelihood mapping methods were calculated using 381 a parametric bootstrap.

382 
384 To simulate alignments with introgression, we extended the two-tree simulation approach

385 described in Posada and Crandall (2002) and shown in Figure 6. This method uses forward

386 time phylogenetic simulations to simulate alignments which mimic those that would be

387 produced by introgression events and allows for control over the placement and timing and

388 of introgression events. In principle, one alignment can be simulated along a tree and then a

389 portion of DNA from one species replaced by a portion of DNA from a second species. Here,

390 we achieve the same results by simulating DNA along two trees and then concatenating the

391 two sequences to mimic the result of introgression. This approach provides a simple and

392 flexible framework for simulating introgression on multiple sequence alignments (Posada

393 and Crandall 2002).

395 We used this framework to simulate datasets under two scenarios of varying treelikeness:

396 increasing proportion of introgressed DNA and increasing number of introgression events.

397 Firstly, we investigated the effect of increasing the proportion of introgressed DNA (i.e.

398 proportion of final tree) by simulating sequence alignments on a balanced 8-taxon tree from

$3990-50 \%$ introgressed DNA sequence in $1 \%$ intervals. 10 replicates were conducted for each

400 set of simulation parameters. We applied the following test statistics to each alignment:

401 tree proportion, $\delta$ plots, likelihood mapping, PHI test and 3SEQ. Due to the high

402 computational expense of the parametric bootstrap, our tree proportion test was calculated

403 only for the proportion of introgressed DNA from $0 \%-50 \%$ in $10 \%$ intervals ( 6 intervals

404 total). Secondly, we investigated the effect of increasing the number of introgression events

405 by simulating 0 to 8 introgression events on a 32-taxon balanced tree. A 32-taxon balanced

406 tree has 8 balanced subtrees, each consisting of two clades with two species each. Each

407 introgression event takes place within one subtree, allowing from 0 to 8 simultaneous

408 events. For this set of simulations, we fixed the proportion of introgressed DNA at $50 \%$. We

409 performed 100 replicates of each set of simulation parameters. Five test statistics were

410 applied to each alignment as above. The tree proportion test was only performed for the

411 first ten replicates of each set of simulation parameters due to the high computational load.

413 Other simulation parameters were as follows. All simulations were repeated for reciprocal

414 and non-reciprocal introgression events. A non-reciprocal event, in which DNA is

415 introgressed unidirectionally from one lineage into another, is shown in Figure 6. In a 
416 reciprocal event, there is a bidirectional exchange of genetic material between two species.

417 For all simulations, we fixed the sequences length to 1300 base pairs (the average length of

418 a transcript in eukaryotes, from Xu et al. (2006)), and the model of substitution to the Jukes-

419 Cantor model (Jukes and Cantor 1969). We simulated four substitution rates (in

420 substitutions per site), $0.05,0.1,0.5$ and 1 , to simulate varying rates of molecular evolution

421 across simulations. The total number of simulated alignments was 4080 for the first set of

422 simulations and 6800 for the second set.

423

424 The scripts for this analysis were written in R v3.6.3 (R Core Team 2020) using the packages

425 ape v5.4-1 (Paradis et al. 2004), ggplot2 v3.3.2 (Wickham 2016), phangorn v2.5.5 (Schliep

426 2011), phytools v0.7-20 (Revell 2012), seqinr v3.6.1 (Charif and Lobry 2007) , stringr v1.4.0

427 (Wickham 2019) and TreeSim v2.4 (Stadler 2017). Code to replicate all simulations is 428 available from https://github.com/caitlinch/treelikeness. Results from the simulations

429 containing test statistic and statistical test results for all six tests are available in the article 430 and in its online supplementary material.

\section{Acknowledgements}

433 The authors would like to thank Barbara Holland, Lindell Bromham, David Gordon and Rod 434 Peakall for their comments and advice. This work was supported by the Australian Research 435 Council grant no. DP-200103151 to R.L. and B.Q.M. 
bioRxiv preprint doi: https://doi.org/10.1101/2021.02.16.431544; this version posted February 17, 2021. The copyright holder for this preprint (which was not certified by peer review) is the author/funder, who has granted bioRxiv a license to display the preprint in perpetuity. It is made available under aCC-BY 4.0 International license.

\section{$436 \quad$ Figures}

437 Figure 1

(a)

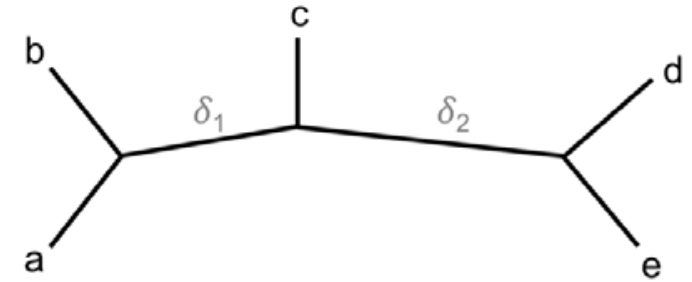

(b)

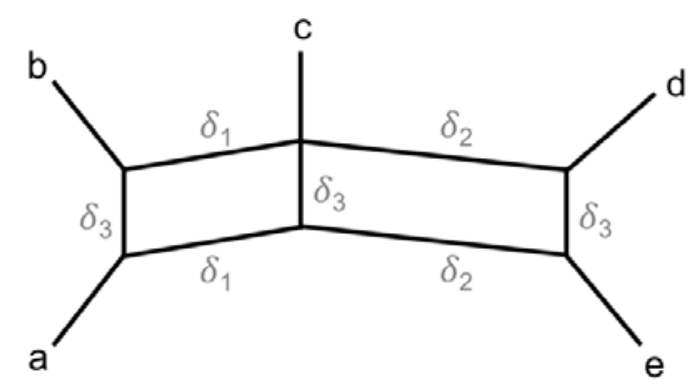

(c)

\begin{tabular}{|ccc|}
\hline Split & Bipartition & Weight $(\lambda)$ \\
\hline$\delta_{1}$ & ab $\mid$ cde & 0.4 \\
\hline$\delta_{2}$ & abc $\mid$ de & 0.6 \\
\hline$\delta_{3}$ & ae $\mid$ bcd & 0.2 \\
\hline
\end{tabular}

(d)

Tree proportion $(T P)=$

$\frac{\lambda\left(\delta_{1}\right)+\lambda\left(\delta_{2}\right)}{\lambda\left(\delta_{1}\right)+\lambda\left(\delta_{2}\right)+\lambda\left(\delta_{3}\right)}$

$T P=\frac{0.4+0.6}{0.4+0.6+0.2}$

$T P=0.83$ 
bioRxiv preprint doi: https://doi org/10.1101/2021.02.16.431544 * this version posted February 17,2021 . The copyright holder for this preprint (which was not certified by peer review) is the author/funder, who has granted bioRxiv a license to display the preprint in perpetuity. It is made available under aCC-BY 4.0 International license.

\section{$440 \quad$ Figure 2}

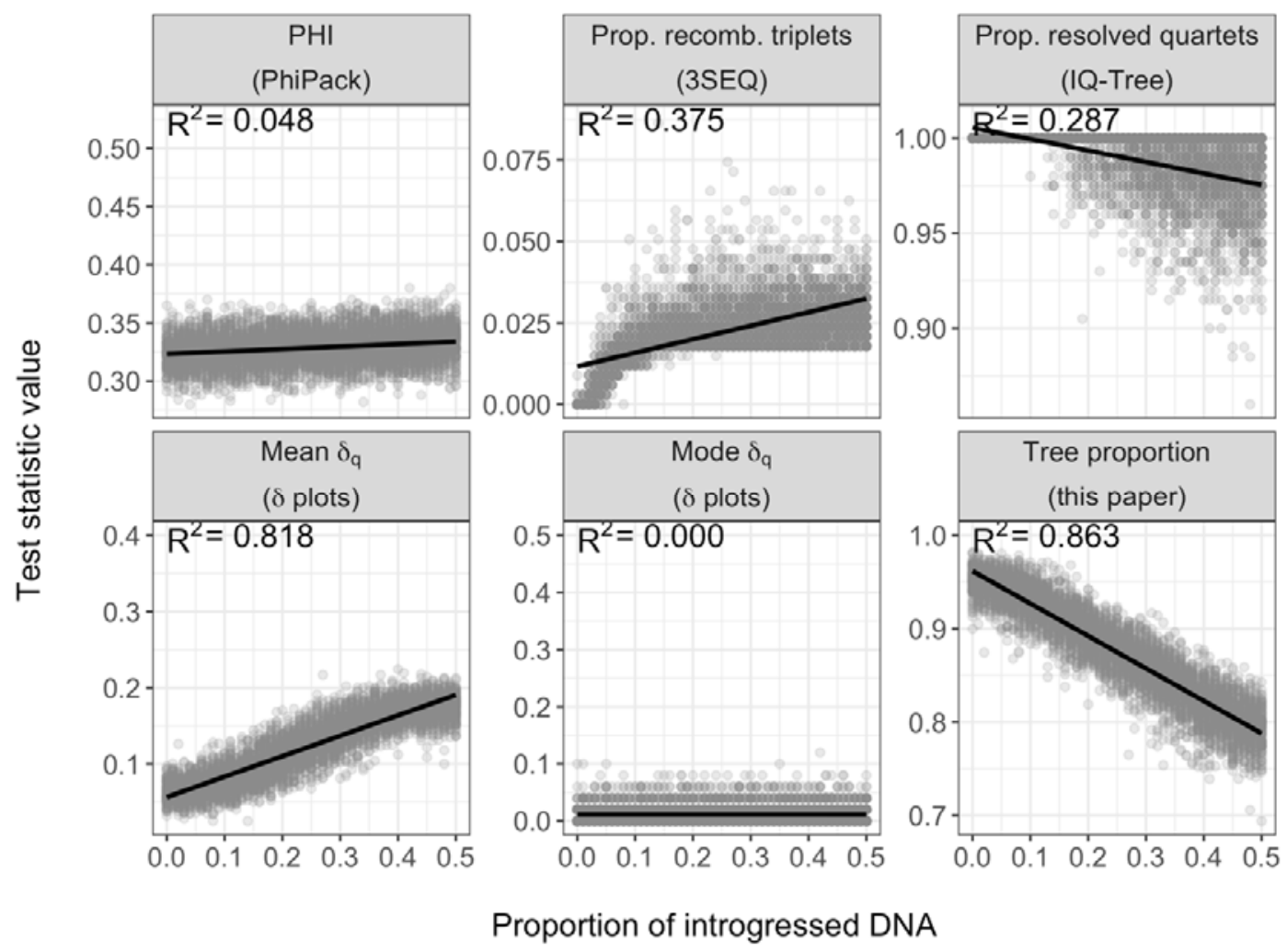


bioRxiv preprint doi: https://doi.org/10.1101/2021.02.16.431544; this version posted February 17, 2021. The copyright holder for this preprint (which was not certified by peer review) is the author/funder, who has granted bioRxiv a license to display the preprint in perpetuity. It is made available under aCC-BY 4.0 International license.

443 Figure 3

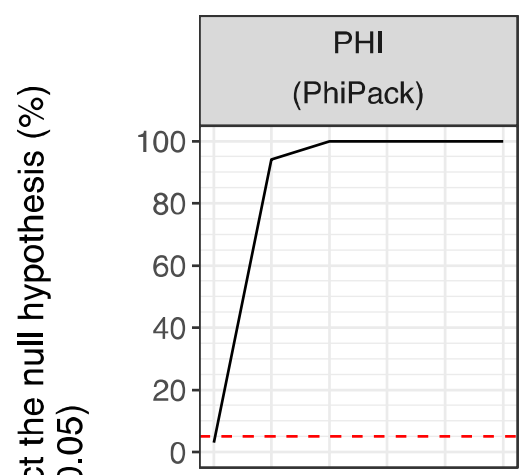

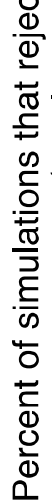

Mean $\delta_{\mathrm{q}}$

( $\delta$ plots)

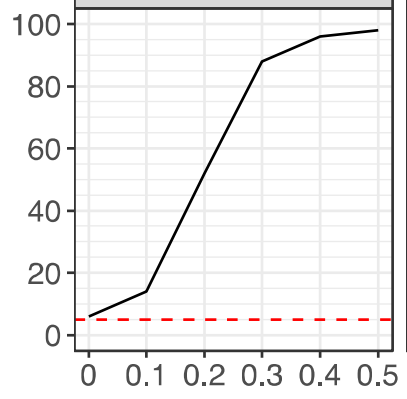

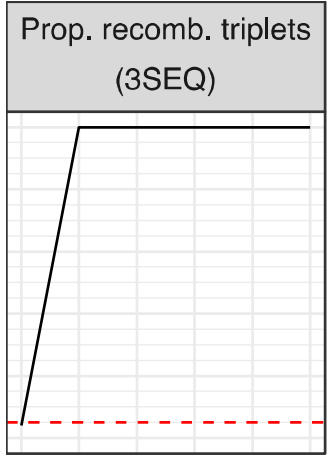
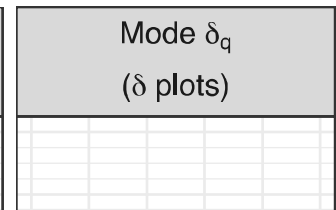
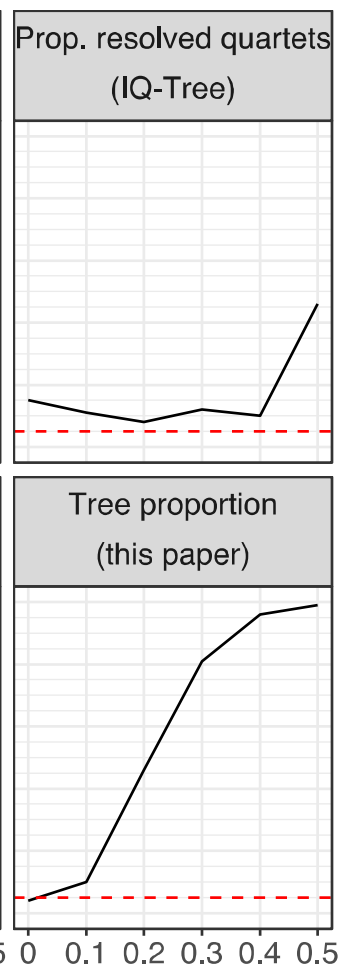

Ideal false

positive rate

_. $5 \%$ when

$a=0.05$

Proportion of introgressed DNA 
bioRxiv preprint doi: https://doi.org/10.1101/2021.02.16.431544: this version posted February 17, 2021. The copyright holder for this preprint (which was not certified by peer review) is the author/funder, who has granted bioRxiv a license to display the preprint in perpetuity. It is made available under aCC-BY 4.0 International license.

\section{$446 \quad$ Figure 4}

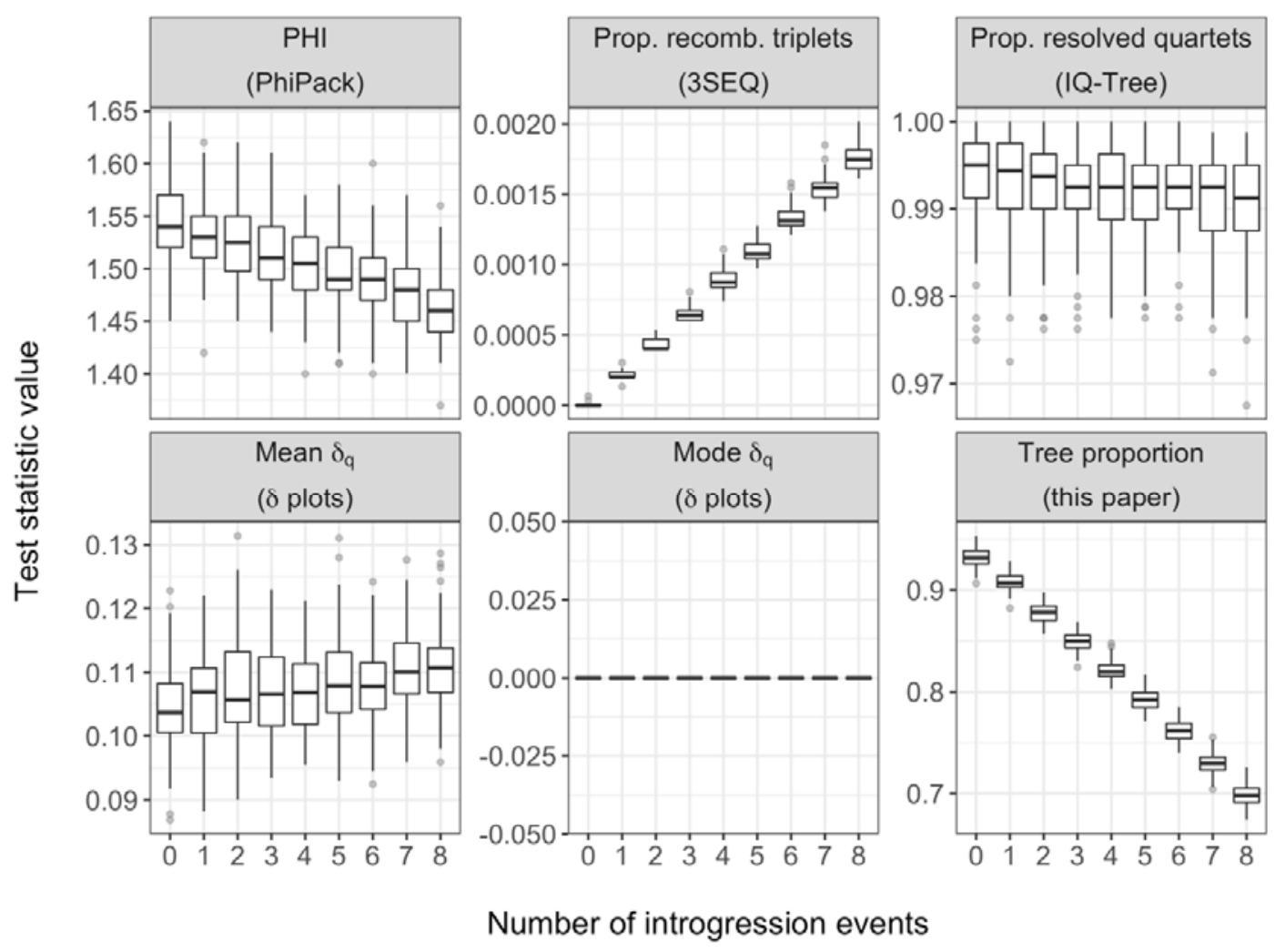

447 
bioRxiv preprint doi: https://doi.org/10.1101/2021.02.16.431544; this version posted February 17, 2021. The copyright holder for this preprint (which was not certified by peer review) is the author/funder, who has granted bioRxiv a license to display the preprint in perpetuity. It is made available under aCC-BY 4.0 International license.

449 Figure 5

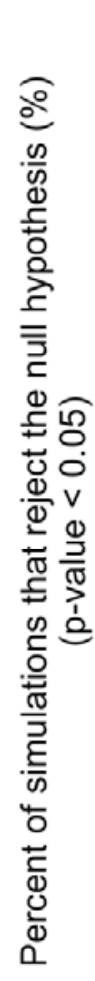
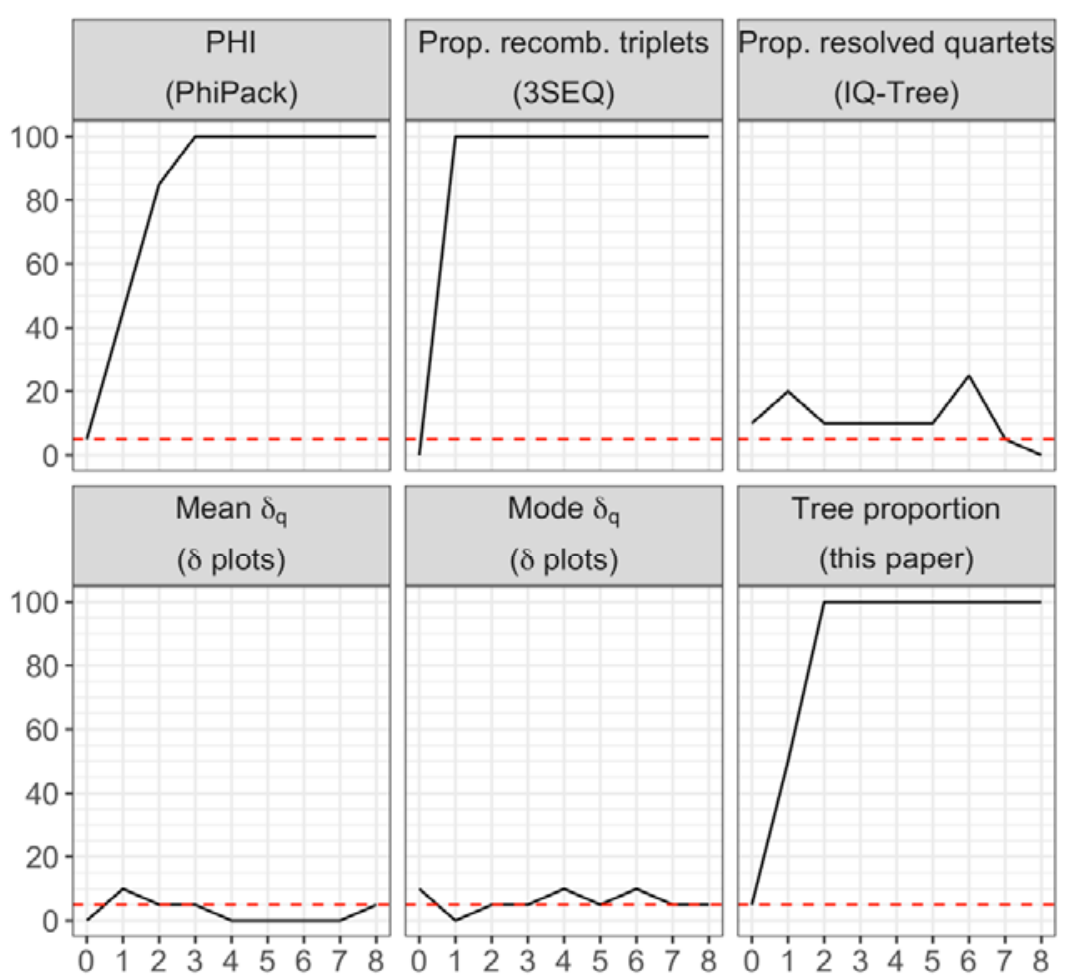

Ideal false

positive rate

- $5 \%$ when

Number of introgression events

450 


\section{Figure 6}

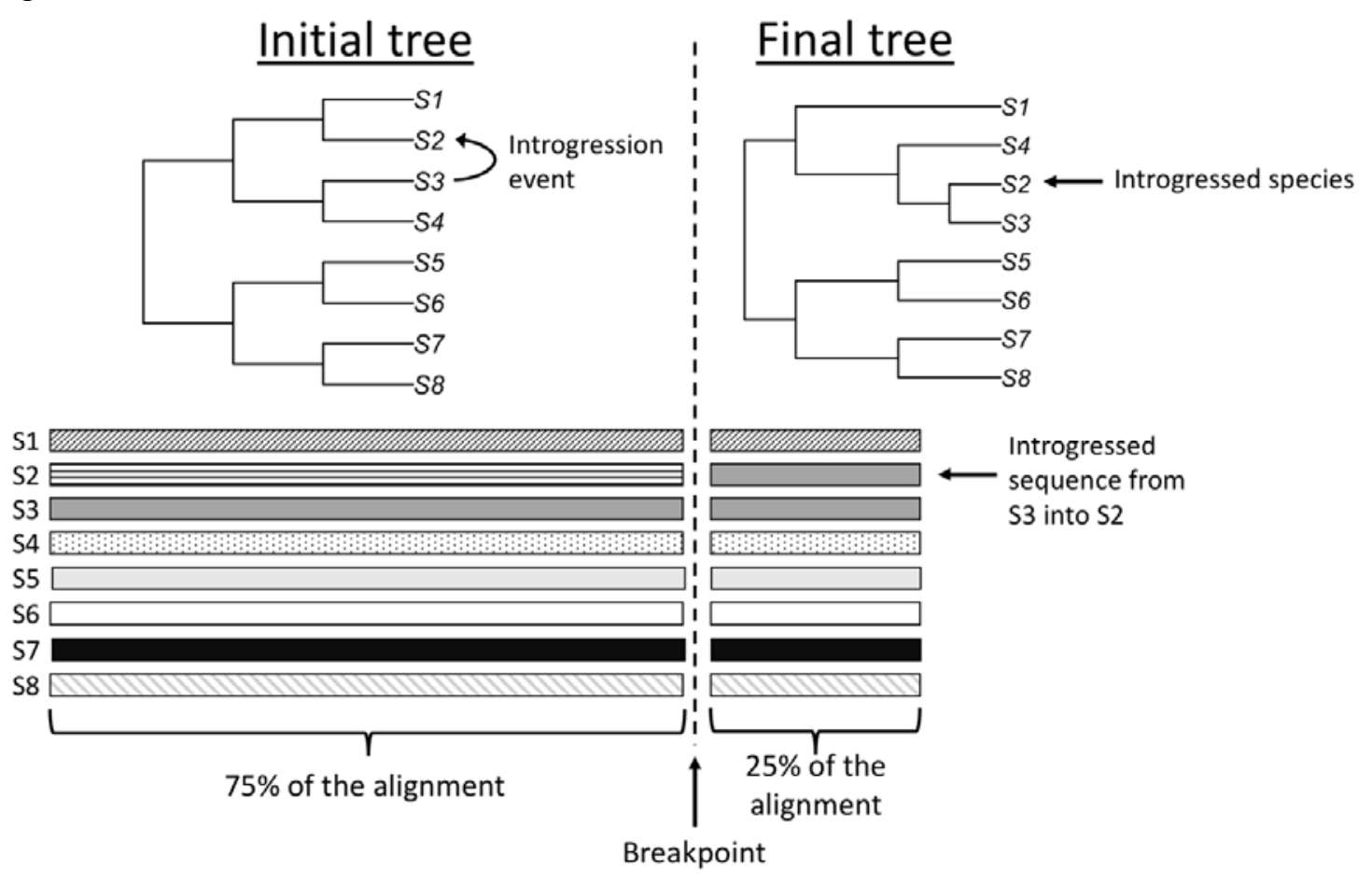

$455 \quad$ Figure Legends

456 Figure 1. a. a simple phylogenetic tree for five taxa. Non-trivial splits are labelled. b. A split 457 phylogenetic network for the same five taxa. Non-trivial splits are labelled. c. Table showing 458 the bipartition of taxa and weight for each non-trivial split. d. Sample calculation of the tree 459 proportion for the alignment with tree $\mathbf{a}$ and network $\mathbf{b}$.

460 Figure 2: Test statistic values for increasing proportions of introgressed DNA from 0 to 0.5 in 4610.01 increments with one close, non-reciprocal introgression events and tree depth of 0.5 (n $462=100$ ). Each point represents the test statistic value for a single simulated alignment. Prop. 463 recomb. triplets is the proportion of recombinant triples. Prop. resolved quartets is the 464 proportion of resolved quartets.

465 Figure 3: Percentage of simulated alignments that reject the null hypothesis as the 466 proportion of introgressed DNA increases for all six test statistics. Simulated alignments had 467 a single close, non-reciprocal introgression event and tree depth was 0.5. Each line 468 represents the number of alignments (out of 100 replicates) that reject the null hypothesis 
469 of treelikeness. Prop. recomb. triplets is the proportion of recombinant triples. Prop.

470 resolved quartets is the proportion of resolved quartets.

471 Figure 4: Test statistic values for number of non-reciprocal introgression events and tree

472 depth of $0.5(n=100)$. Each box shows the distribution of test statistic values at a certain

473 number of introgression events. The whiskers extend to the closest observed value no more

474 than 1.5 times the interquartile range away from the box. Points represent outliers. Prop.

475 recomb. triplets is the proportion of recombinant triples. Prop. resolved quartets is the 476 proportion of resolved quartets.

477 Figure 5: Percentage of simulated alignments that reject the null hypothesis as the number 478 of non-reciprocal introgression events increases for all six test statistics. Simulated 479 alignments had a tree depth of 0.5. Each line represents the number of alignments (out of 480100 replicates) that reject the null hypothesis of treelikeness. The red dotted line represents 481 the ideal false positive rate of $5 \%$ when $\alpha=0.05$. Prop. recomb. triplets is the proportion of 482 recombinant triples. Prop. resolved quartets is the proportion of resolved quartets.

483 Figure 6: Simulation of a mosaic multiple sequence alignment with an introgression event, 484 adapted from Posada and Crandall (2002). The initial sequence ( $n \%$ of the final sequence, 485 here $n=75 \%$ ) is simulated along a balanced 8-taxon tree. The introgression event shown 486 here consists of genetic material from S3 overwriting the original sequence in S2 (as shown 487 by the arrows). In the final tree, the introgression event has occurred, moving the position 488 of S2 in the phylogeny. $25 \%((1-n) \%)$ of the alignment is then simulated along this tree. 489 Two trees are needed to explain the evolutionary history of this alignment, violating the 490 treelikeness assumption.

\section{References}

492 Baric S, Salzburger W, Sturmbauer C. 2003. Phylogeography and evolution of the Tanganyikan Cichlid genus Tropheus based upon mitochondrial DNA Sequences. J. Mol. Evol. 56(1):54-68.

Boni MF, Posada D, Feldman MW. 2007. An exact nonparametric method for inferring mosaic structure in sequence triplets. Genetics 176(2):1035-1047.

Brown JM, Thomson RC. 2018. Evaluating model performance in evolutionary biology. Annu. Rev. Ecol. Evol. Syst. 49(1):95-114.

500

Bruen T. 2005. PhiPack. Online, available at https://www.maths.otago.ac.nz/ dbryant/software.html. 
501

502

503

504

505

506

507

508

509

510

511

512

513

514

515

516

517

518

519

520

521

522

523

524

525

526

527

528

529

530

531

532

533

534

535

536

537

538

539

540

541

542

543

544

545

546

547
Bruen TC, Philippe H, Bryant D. 2006. A simple and robust statistical test for detecting the presence of recombination. Genetics 172(4):2665.

Bryant D, Moulton V. 2004. Neighbor-Net: an agglomerative method for the construction of phylogenetic networks. Mol. Bio. Evol. 21(2):255-265.

Buesa J, Collado B, López-Andújar P, Abu-Mallouh R, Rodríguez Díaz J, García Díaz A, Prat J, Guix S, Llovet T, Prats $G$ et al. . 2002. Molecular epidemiology of caliciviruses causing outbreaks and sporadic cases of acute gastroenteritis in Spain. J. Clin. Microbiol. 40(8):2854-2859.

Cabanne GS, d'Horta FM, Sari EHR, Santos FR, Miyaki CY. 2008. Nuclear and mitochondrial phylogeography of the Atlantic forest endemic Xiphorhynchusfuscus (Aves: Dendrocolaptidae): biogeography and systematics implications. Mol. Phylogenet. Evol. 49(3):760-773.

Charif D, Lobry JR. 2007. SeqinR 1.0-2: a contributed package to the R project for statistical computing devoted to biological sequences retrieval and analysis. In: Bastolla $U$, Porto M, Roman HE, Vendruscolo M, editors. Structural approaches to sequence evolution: Molecules, networks, populations. New York: Springer Verlag. p. 207-232.

Coiro M, Barone Lumaga MR. 2018. Disentangling historical signal and pollinator selection on the micromorphology of flowers: an example from the floral epidermis of the Nymphaeaceae. Plant Biol. 20(5):902-915.

Croll D, Sanders IR. 2009. Recombination in Glomus intraradices, a supposed ancient asexual arbuscular mycorrhizal fungus. BMC Evol. Biol. 9(1):13.

Croucher NJ, Page AJ, Connor TR, Delaney AJ, Keane JA, Bentley SD, Parkhill J, Harris SR. 2015. Rapid phylogenetic analysis of large samples of recombinant bacterial whole genome sequences using Gubbins. Nucleic Acids Res. 43(3):e15.

Cui R, Schumer M, Kruesi K, Walter R, Andolfatto P, Rosenthal GG. 2013. Phylogenomics reveals extensive reticulate evolution in Xiphophorus fishes. Evolution 67(8):21662179.

D'Horta FM, Cabanne GS, Meyer D, Miyaki CY. 2011. The genetic effects of Late Quaternary climatic changes over a tropical latitudinal gradient: diversification of an Atlantic Forest passerine. Mol. Ecol. 20(9):1923-1935.

Dashper SG, Mitchell HL, Seers CA, Gladman SL, Seemann T, Bulach DM, Chandry PS, Cross KJ, Cleal SM, Reynolds EC. 2017. Porphyromonas gingivalis uses specific domain rearrangements and allelic exchange to generate diversity in surface virulence factors. Front. Microbiol. 8:48.

de Souza WM, Fumagalli MJ, de Araujo J, Sabino-Santos G, Maia FGM, Romeiro MF, Modha S, Nardi MS, Queiroz LH, Durigon EL et al. . 2018. Discovery of novel anelloviruses in small mammals expands the host range and diversity of the Anelloviridae. Virology 514:9-17.

Dress AWM. 1984. Trees, tight extensions of metric spaces, and the cohomological dimension of certain groups: a note on combinatorial properties of metric spaces Adv. Math. 53:321-402.

Eigen M, Winkler-Oswatitsch R, Dress A. 1988. Statistical geometry in sequence space: a method of quantitative comparative sequence analysis. Proc. Natl. Acad. Sci. U.S.A. 85(16):5913.

Elena SF, Dopazo J, de la Peña M, Flores R, Diener TO, Moya A. 2001. Phylogenetic analysis of viroid and viroid-like satellite RNAs from plants: a reassessment. J. Mol. Evol. 53(2):155-159. 
548

549

550

551

552

553

554

555

556

557

558

559

560

561

562

563

564

565

566

567

568

569

570

571

572

573

574

575

576

577

578

579

580

581

582

583

584

585

586

587

588

589

590

591

592

593

594

Faria NR, Azevedo RdSdS, Kraemer MUG, Souza R, Cunha MS, Hill SC, Thézé J, Bonsall MB, Bowden TA, Rissanen I et al. . 2016. Zika virus in the Americas: early epidemiological and genetic findings. Science 352(6283):345-349.

Goldman N. 1993. Statistical tests of models of DNA substitution. J. Mol. Evol. 36(2):182198.

Grimm GW, Renner SS. 2013. Harvesting Betulaceae sequences from GenBank to generate a new chronogram for the family. Bot. J. Linn. Soc. 172(4):465-477.

Grismer LL, Wood JRPL, Quah ESH, Anuar S, Ngadi EB, Izam NAM, Ahmad N. 2018.

Systematics, ecomorphology, cryptic speciation and biogeography of the lizard genus Tytthoscincus Linkem, Diesmos \& Brown (Squamata: Scincidae) from the sky-island archipelago of Peninsular Malaysia. Zool. J. Linn. Soc. 183(3):635-671.

Grybchuk D, Akopyants NS, Kostygov AY, Konovalovas A, Lye L-F, Dobson DE, Zangger H, Fasel N, Butenko A, Frolov AO et al. . 2018. Viral discovery and diversity in trypanosomatid protozoa with a focus on relatives of the human parasite Leishmania. Proc. Natl. Acad. Sci. U.S.A. 115(3):E506.

Harris SR, Clarke IN, Seth-Smith HMB, Solomon AW, Cutcliffe LT, Marsh P, Skilton RJ, Holland MJ, Mabey D, Peeling RW et al. . 2012. Whole-genome analysis of diverse Chlamydia trachomatis strains identifies phylogenetic relationships masked by current clinical typing. Nat. Genet. 44:413.

Haubold B, Krause L, Horn T, Pfaffelhuber P. 2013. An alignment-free test for recombination. Bioinformatics 29(24):3121-3127.

Holland BR, Huber KT, Dress A, Moulton V. 2002. $\delta$ plots: a tool for analyzing phylogenetic distance data. Mol. Biol. Evol. 19(12):2051-2059.

Huson DH, Bryant D. 2006. Application of phylogenetic networks in evolutionary studies. Mol. Bio. Evol. 23(2):254-267. Software available from www.splitstree.org.

Huson DH, Rupp R, Scornavacca C. 2010. Phylogenetic networks. Cambridge: Cambridge University Press.

Joly S, Bruneau A. 2006. Incorporating allelic variation for reconstructing the evolutionary history of organisms from multiple genes: an example from Rosa in North America. Syst. Biol. 55(4):623-636.

Jukes TH, Cantor CR. 1969. Evolution of protein molecules. In: Munro HN, editor. Mammalian Protein Metabolism. New York: Academic Press. p. 21-132.

Kalyaanamoorthy S, Minh BQ, Wong TKF, von Haeseler A, Jermiin LS. 2017. ModelFinder: fast model selection for accurate phylogenetic estimates. Nat. Methods 14(6):587589.

Kang YJ, Kim SK, Kim MY, Lestari P, Kim KH, Ha B-K, Jun TH, Hwang WJ, Lee T, Lee J et al. . 2014. Genome sequence of mungbean and insights into evolution within Vigna species. Nat. Commun. 5:5443.

Kennedy M, Holland BR, Gray RD, Spencer HG. 2005. Untangling long branches: identifying conflicting phylogenetic signals using spectral analysis, Neighbor-Net, and consensus networks. Syst. Biol. 54(4):620-633.

Kozak KM, Wahlberg N, Neild AFE, Dasmahapatra KK, Mallet J, Jiggins CD. 2015. Multilocus species trees show the recent adaptive radiation of the mimetic Heliconius butterflies. Syst. Biol. 64(3):505-524.

Kück P, Meusemann K, Dambach J, Thormann B, von Reumont BM, Wägele JW, Misof B. 2010. Parametric and non-parametric masking of randomness in sequence alignments can be improved and leads to better resolved trees. Front. Zool. 7(1):10. 
595

596

597

598

599

600

601

602

603

604

605

606

607

608

609

610

611

612

613

614

615

616

617

618

619

620

621

622

623

624

625

626

627

628

629

630

631

632

633

634

635

636

637

638

639

640
Lam HM, Ratmann O, Boni MF. 2018. Improved algorithmic complexity for the 3SEQ recombination detection algorithm. Mol. Biol. Evol. 35(1):247-251.

Lei M, Dong D. 2016. Phylogenomic analyses of bat subordinal relationships based on transcriptome data. Sci. Rep. 6:27726.

Lemmon AR, Moriarty EC. 2004. The importance of proper model assumption in Bayesian phylogenetics. Syst. Biol. 53(2):265-277.

Li X, Liu H, Liu L, Feng Y, Kalish ML, Ho SYW, Shao Y. 2017. Tracing the epidemic history of HIV-1 CRF01_AE clusters using near-complete genome sequences. Sci. Rep. 7(1):4024.

Mallet J, Besansky N, Hahn MW. 2016. How reticulated are species? Bioessays 38(2):140149.

Meier-Kolthoff JP, Göker M. 2019. TYGS is an automated high-throughput platform for state-of-the-art genome-based taxonomy. Nat. Commun. 10(1):2182.

Mendes F, K., Livera A, P., Hahn M, W. 2019. The perils of intralocus recombination for inferences of molecular convergence. Philos. Trans. R. Soc. B. 374(1777):20180244.

Minh BQ, Schmidt HA, Chernomor O, Schrempf D, Woodhams MD, von Haeseler A, Lanfear R. 2020. IQ-TREE 2: new models and efficient methods for phylogenetic inference in the genomic era. Mol. Biol. Evol. 37(5):1530-1534.

Morgan CC, Creevey CJ, O'Connell MJ. 2014. Mitochondrial data are not suitable for resolving placental mammal phylogeny. Mamm. Genome. 25(11):636-647.

Nadan S, Walter JE, Grabow WOK, Mitchell DK, Taylor MB. 2003. Molecular characterization of astroviruses by reverse transcriptase PCR and sequence analysis: comparison of clinical and environmental isolates from South Africa. Appl. Environ. Microb. 69(2):747.

Naser-Khdour S, Minh BQ, Zhang W, Stone EA, Lanfear R. 2019. The prevalence and impact of model violations in phylogenetic analysis. Genome Biol. Evol. 11(12):3341-3352.

Ogura Y, Ooka T, Iguchi A, Toh H, Asadulghani M, Oshima K, Kodama T, Abe H, Nakayama K, Kurokawa $\mathrm{K}$ et al. . 2009. Comparative genomics reveal the mechanism of the parallel evolution of 0157 and non-0157 enterohemorrhagic Escherichia coli. Proc. Natl. Acad. Sci. U.S.A. 106(42):17939.

Paradis E, Claude J, Strimmer K. 2004. APE: analyses of phylogenetics and evolution in R language. Bioinformatics 20(2):289-290.

Pearce SL, Clarke DF, East PD, Elfekih S, Gordon KHJ, Jermiin LS, McGaughran A, Oakeshott JG, Papanikolaou A, Perera OP et al. . 2017. Genomic innovations, transcriptional plasticity and gene loss underlying the evolution and divergence of two highly polyphagous and invasive Helicoverpa pest species. BMC Biol. 15(1):63.

Penny D, Hendy MD, Steel MA. 1992. Progress with methods for constructing evolutionary trees. Trends Ecol. Evol. 7(3):73-79.

Pinho C, Harris DJ, Ferrand N. 2008. Non-equilibrium estimates of gene flow inferred from nuclear genealogies suggest that Iberian and North African wall lizards (Podarcis spp.) are an assemblage of incipient species. BMC Evol. Biol. 8(1):63.

Pitra C, Lieckfeldt D, Frahnert S, Fickel J. 2002. Phylogenetic relationships and ancestral areas of the bustards (Gruiformes: Otididae), inferred from mitochondrial DNA and nuclear intron sequences. Mol. Phylogenet. Evol. 23(1):63-74.

Posada D, Crandall KA. 2002. The effect of recombination on the accuracy of phylogeny estimation. J. Mol. Evol. 54(3):396-402. 
641 R Core Team. 2020. R: A Language and Environment for Statistical Computing. Vienna,

642

643

644

645

646

647

648

649

650

651

652

653

654

655

656

657

658

659

660

661

662

663

664

665

666

667

668

669

670

671

672

673

674

675

676

677

678

679

680

681

682

683

684

685

686

687 Austria: R Foundation for Statistical Computing.

Revell LJ. 2012. phytools: An R package for phylogenetic comparative biology (and other things). Methods Ecol. Evol. 3:217-223.

Salemi M, Desmyter J, Vandamme AM. 2000. Tempo and mode of human and simian Tlymphotropic virus (HTLV/STLV) evolution revealed by analyses of full-genome sequences. Mol. Biol. Evol. 17(3):374-386.

Salzburger W, Martens J, Sturmbauer C. 2002. Paraphyly of the Blue Tit (Parus caeruleus) suggested from cytochrome b sequences. Mol. Phylogenet. Evol. 24(1):19-25.

Schliep KP. 2011. phangorn: phylogenetic analysis in R. Bioinformatics 27(4):592-592.

Scornavacca C, Galtier N. 2017. Incomplete lineage sorting in mammalian phylogenomics. Syst. Biol. 66(1):112-120.

Shi CM, Yang Z. 2018. Coalescent-based analyses of genomic sequence data provide a robust resolution of phylogenetic relationships among major groups of gibbons. Mol. Biol. Evol. 35(1):159-179.

Shi W, Freitas IT, Zhu C, Zheng W, Hall WW, Higgins DG. 2012. Recombination in hepatitis C virus: identification of four novel naturally occurring inter-subtype recombinants. PloS one 7(7):e41997-e41997.

Short DPG, O'Donnell K, Geiser DM. 2014. Clonality, recombination, and hybridization in the plumbing-inhabiting human pathogen Fusarium keratoplasticum inferred from multilocus sequence typing. BMC Evolutionary Biology 14(1):91.

Song H, Sheffield NC, Cameron SL, Miller KB, Whiting MF. 2010. When phylogenetic assumptions are violated: base compositional heterogeneity and among-site rate variation in beetle mitochondrial phylogenomics. Syst. Entomol. 35(3):429-448.

Stadler T. 2017. TreeSim: simulating phylogenetic trees. R package version 2.4 . http://CRAN.R-project.org/package=TreeSim

Stanborough T, Fegan N, Powell SM, Singh T, Tamplin M, Chandry PS. 2018. Genomic and metabolic characterization of spoilage-associated Pseudomonas species. Int. J. Food Microbiol. 268:61-72.

Steiner G, Dreyer H. 2003. Molecular phylogeny of Scaphopoda (Mollusca) inferred from 18S rDNA sequences: support for a Scaphopoda-Cephalopoda clade. Zool. Scr. 32(4):343356.

Strimmer K, von Haeseler A. 1997. Likelihood-mapping: a simple method to visualize phylogenetic content of a sequence?alignment. Proc. Natl. Acad. Sci. U.S.A. 94(13):6815-6819.

Tay WT, Walsh TK, Downes S, Anderson C, Jermiin LS, Wong TKF, Piper MC, Chang ES, Macedo IB, Czepak C et al. . 2017. Mitochondrial DNA and trade data support multiple origins of Helicoverpa armigera (Lepidoptera, Noctuidae) in Brazil. Sci. Rep. 7:45302.

Tian CF, Zhou YJ, Zhang YM, Li QQ, Zhang YZ, Li DF, Wang S, Wang J, Gilbert LB, Li YR et al. . 2012. Comparative genomics of rhizobia nodulating soybean suggests extensive recruitment of lineage-specific genes in adaptations. Proc. Natl. Acad. Sci. U.S.A. 109(22):8629.

Verbruggen $\mathrm{H}$, Theriot EC. 2008. Building trees of algae: some advances in phylogenetic and evolutionary analysis. Eur. J. Phycol. 43(3):229-252.

Weinert LA, Werren JH, Aebi A, Stone GN, Jiggins FM. 2009. Evolution and diversity of Rickettsia bacteria. BMC Biol. 7(1):6. 
688

689

690

691

692

693

694

695

696

697

698

699

700

701

702

703

704

705

706

707

708

709

710

711

712
Weisrock DW, Smith SD, Chan LM, Biebouw K, Kappeler PM, Yoder AD. 2012. Concatenation and concordance in the reconstruction of mouse lemur phylogeny: an empirical demonstration of the effect of allele sampling in phylogenetics. Mol. Biol. Evol. 29(6):1615-1630.

White DJ, Bryant D, Gemmell NJ. 2013. How good are indirect tests at detecting recombination in human mtDNA? G3 (Bethes da) 3(7):1095-1104.

White DJ, Gemmell NJ. 2009. Can indirect tests detect a known recombination event in human mtDNA? Mol. Biol. Evol. 26(7):1435-1439.

Wickham H. 2016. ggplot2: Elegant Graphics for Data Analysis. New York: Springer-Verlag. Wickham H. 2019. stringr: Simple, Consistent Wrappers for Common String Operations. R package version 1.4.0. https://CRAN.R-project.org/package=stringr.

Wielstra B, Arntzen JW, van der Gaag KJ, Pabijan M, Babik W. 2014. Data concatenation, Bayesian concordance and coalescent-based analyses of the species tree for the rapid radiation of Triturus newts. PLOS ONE 9(10):e111011.

Wiens JJ. 1998. Combining data sets with different phylogenetic histories. Syst. Biol. 47(4):568-581.

Wu M, Kostyun JL, Hahn MW, Moyle LC. 2018. Dissecting the basis of novel trait evolution in a radiation with widespread phylogenetic discordance. Mol. Ecol. 27(16):3301-3316.

Xu L, Chen H, Hu X, Zhang R, Zhang Z, Luo ZW. 2006. Average gene length is highly conserved in prokaryotes and eukaryotes and diverges only between the two kingdoms. Mol. Biol. Evol. 23(6):1107-1108.

Zhao L, Li X, Zhang N, Zhang S-D, Yi T-S, Ma H, Guo Z-H, Li D-Z. 2016. Phylogenomic analyses of large-scale nuclear genes provide new insights into the evolutionary relationships within the rosids. Mol. Phylogenet. Evol. 105:166-176. 